\title{
Machine-Independent Complexity Theory
}

\author{
Joel I. Seiferas \\ Computer Science Department \\ University of Rochester \\ Rochester, New York, U. S. A. 14627 \\ joel@cs.rochester.edu
}

January 20, 1989

\begin{abstract}
This chapter provides an accessible and straightforward tour through some of the most basic notions and remarkable phenomena in machine-independent computational complexity. It includes versions of the recursion, compression, speedup, gap, union, and honesty theorems. Where possible, the results are derived from machine-dependent ones, in the particularly convenient concrete setting of space-bounded Turing machines.
\end{abstract}

\section{Introduction}

The familiar measures of computational complexity are time and space. Generally speaking, time is the number of discrete steps in a computation, and space is the number of distinct storage locations accessed by the instructions of the computation.

What we would like to classify is the computational complexity of an entire problem; but a problem has a countably infinite number of "instances", and may be solved in many different ways, on many different computer architectures. Since an overall solution has a time complexity and a space complexity for each instance, we can specify its complexity as a function on problem instances, or, for more gross purposes, on some measure of the "size" of problem instances. To specify the computational time or space used by a partial solution, we can use a partial function.

Each separate solution to a problem will have its own computational complexity. What should be the time or space complexity of the problem? The natural desire is to choose the smallest such complexity as the inherent one. A set of complexities, however, since they are functions and not mere members of the set $N$ of natural numbers, need not necessarily have a smallest member; there might be "trade-offs", or even infinite descending chains. If no one solution for a problem does have the least complexity, we say that the problem "has speedup", basing our terminology on the special case of time complexity. We show in this chapter that there really are computational problems with arbitrarily dramatic speedup; and we show, on the other hand, that there are problems without it (i.e., that do have essentially optimum solutions). Moreover, we show that the existence of these phenomena is "machine independent", depending neither on the choice of complexity measure nor on the choice of computer architecture, within reason.

Another natural, but very different, notion of complexity is program size [4]. Program size is just a single natural number, so a problem does have a well-defined 
best complexity of this static variety, at least for a particular, fixed computer architecture. It turns out that the essential features of this notion of complexity are again "machine independent" $[4,13]$. What is more remarkable, however, is the drastic inherent trade-offs that can exist between program size and dynamic computational complexity [24].

Although we are interested in machine-independent phenomena, we will start in a very specific, concrete setting. Our results in this setting can be viewed initially as mere illuminating examples. In the end, however, we will derive most of our machine-independent results as corollaries of these machine-dependent ones, a method first used by Hartmanis and Hopcroft [10]. There is an alternative approach based on Manuel Blum's two simple axioms [3] (see below), that can be viewed as machine-independent from the outset. For more complete coverage of both approaches, and for many additional research directions, the reader is encouraged to consult additional surveys, overviews, and research literature. The recent presentations in [7] and [29] are additional good sources of pointers into the literature to date.

\section{Simple Turing Machines, and Space Complexity}

The machines we consider each consist of a finite-state program with access to an input tape and a single storage tape. Recorded on the input tape is an input word, a nonnull, finite string of characters from some finite input alphabet. (If $\Sigma$ is the alphabet, then $\Sigma^{+}$denotes the set of all such strings.) Recorded on the storage tape is a string of characters from the fixed, binary alphabet $\{0,1\}$. The initial content of the storage tape is the trivial word ' 0 ', of length 1. (In general, we denote the length of a word $x$ by $|x|$.) A separate tape head is maintained in some position, initially the leftmost one, on each of the two tapes. The finitestate program consists of a finite set of (control) states, one of which is designated as the initial one, and a finite function indicating how each next computational "action" depends on the visible "display" of the current total state of the entire machine. (In this chapter, we consider only deterministic automata; so we assume each next action is fully determined by the current display.) The display consists of the current control state of the program, the symbol being "scanned" by each tape head, and indication whether each tape head is scanning its tape's leftmost or rightmost symbol. Each action can change the control state and also do any of the following: write 0 or 1 over the scanned symbol on the storage tape; shift a tape head left or right, respectively, if it is not already at the left or right end of its tape; and append an additional 0 onto the right end of the storage tape, if the head is currently at that end. (Because of this special role for 0 , we sometimes refer to it as the "blank" storage tape symbol.) Note that the machine cannot modify or extend its input tape, but that it can modify its storage tape and extend it arbitrarily far to the right. Let us call such a machine an STM (for "simple Turing machine").

From the informal description above, it should be clear how an STM starts and runs on an arbitrary word over its input alphabet. To view it as computing some function or partial function of its input, we must adopt some output convention. For a finite-valued function, we could specify a distinct "final" control state for each possible value, and say that the computed value is the one (if any) whose corresponding final state is first entered. For a more general function, the convention could be to leave the output value encoded somehow at the left end of the storage tape if and when a designated final state is first entered. 
Under the output conventions suggested above, we can view computations as ending when they first enter final states, since subsequent steps do not affect their output. This finally leads to a definition of space complexity: The space $\operatorname{Space}_{M}(x)$ used by STM $M$ on input $x$ is the length of $M$ 's storage tape if and when its computation on that input ends. Space ${ }_{M}(x)$ is undefined if the computation by $M$ on input $x$ does not end. We denote this lack of definition by $\operatorname{Space}_{M}(x)=\infty$, even though this makes it look like $\operatorname{Space}_{M}(x)$ is defined, and even though a nonterminating computation might not ever use much space (if it is in a "loop"). (Again the terminology follows time complexity more closely: A nonterminating computation does consume infinite time.) A benefit is that our implicit conventions regarding arithmetic and comparisons when some of the operands might be undefined are consistent with viewing $\infty$ as "the value infinity".

Actually, it is safe to ignore looping (nonterminating computation on bounded space). From each STM that might loop, we can construct an equivalent one that does not (equivalent in the sense that it computes the same partial function, in exactly the same space). Moreover, the nonlooping STM's obtained in this way are easily recognizable, syntactically. For a description of this construction, see [26].

Our first example of the speedup phenomenon is a simple but universal one: The space complexity of any solution to any problem can be reduced by any constant $c$; i.e., whatever an STM can do in space $S(x)$, another STM can do in space $S^{\prime}(x)=\max (1, S(x)-c)$. By induction, it suffices to prove this for $c=1$.

Proposition (additive-constant speedup). Whatever an $S T M M$ can do in space $S(x)$, another $S T M M^{\prime}$ can do in space $S^{\prime}(x)=\max (1, S(x)-1)$.

Proof: The idea of the simulation is for $M^{\prime}$ to maintain the one leftmost bit of $M$ 's storage tape in its finite control, and to maintain only the rest of $M$ 's storage tape, including head position, on its own storage tape. Of course exceptions must be made until the length of $M$ 's storage tape reaches 2 , and whenever that tape's head scans its leftmost symbol. At each successive instant, the control state of $M^{\prime}$ will consist of the current control state of $M$, the leftmost bit of $M$ 's storage tape, indication whether $M$ 's storage tape has yet reached length 2 , and indication whether $M$ 's storage tape head currently scans the leftmost symbol on its tape. It is inductively straightforward for $M^{\prime}$ to maintain all this and simultaneously to determine and simulate the successive transitions of $M$.

\section{Recursion, Padding, and Compression}

The second phenomenon is sometimes referred to as "compression". Since the additive-constant speedup phenomenon above is universal, we cannot expect to find a problem whose optimum complexity is completely compressed down to a single, precise space bound. It turns out, however, that additive-constant speedup is the only such concession we have to make.

So that we can conveniently ignore additive-constant speedup, we define a slightly "blurred" partial order $\preceq$ on functions and partial functions, so that $f(x) \preceq$ $g(x)$ holds if and only if there is some additive constant $c$ such that, for every $\bar{x}$, $f(x) \leq c+g(x)$. In these terms, we have seen that we cannot hope to avoid speedup from $S(x)$ to $S^{\prime}(x)$ if $S^{\prime}(x) \succeq S(x)$.

Compression Theorem for STM space. Above any computable function, there is a computable space bound $S: \Sigma^{+} \rightarrow N$ for which there is a predicate $P$ : 
$\Sigma^{+} \rightarrow\{0,1\}$, computable within space $S(x)$, but not computable within space $S^{\prime}(x)$ unless $S^{\prime}(x) \succeq S(x)$.

Before we turn to the proof itself, let us introduce some additional technical machinery, notation, and terminology. The proof will involve diagonalization by an STM over other STM's, so we will need a way for an STM to represent other STM's on its tapes. Straightforward formalization and binary encoding of our informal definition of STM's computing partial functions from $\Sigma^{+}$to $\{0,1\}$ yields an easily decodable binary string, or "program", describing each such machine. For each binary string $e$, let $M_{e}$ be the machine described by $e$. (Not every binary string will describe an (explicitly nonlooping) STM via our formalization; but the ones that do not will be easily recognizable, and we adopt the convention that each of these does implicitly "describe" some one fixed machine of our choice.) Let $\varphi_{e}(x)$ denote the partial function computed by $M_{e}$, and let $S_{e}(x)=$ Space $_{M_{e}}(x)$. (Recall that the latter is defined on precisely the same domain as the former.) Finally, say that a total space bound $S: \Sigma^{+} \rightarrow N$ is constructible if there is an STM that, on each input string $x$, extends its storage tape to length exactly $S(x)$, zeros it out, and halts, having "constructed" or "laid out" space $S(x)$. For example, $n^{3}, 2^{n}, n !$, $\left\lfloor n^{2 / 3}\right\rfloor$, and all other common superlogarithmic functions of $n=|x|$ turn out to be constructible, including all the ones in Grzegorczyk's class $\mathcal{E}^{2}$ [21, Section 4].

Several of our proofs will involve procedures that "invoke themselves recursively". Implementation of such procedures is easiest and most transparent on "stored program" computers. Now that we do see how to develop the notion of a machine's "program", let us go back and slightly revise our STM model to include a "stored program" feature. First, we syntactically add one new "action" to the original definition. Our intention is for the new action to involve writing the next bit of the machine's own program code, if there is a next one, over the scanned symbol on the storage tape. With just the syntactic definition, we can already proceed to assign program codes as outlined above; and, having done so, we are able finally to complete the semantic part of the definition in the intended way. Noting that we can easily transform an STM of either variety into an equivalent one of the other variety, we may as well adopt the enhanced version as our standard.

To facilitate the implementation of even mutual recursion, we place one more, explicit but harmless, constraint on the assignment of program codes: At the end of every well-formed code, there should be a clearly delimited block of "unreachable code", more appropriately viewed as a "data segment". To design a pair of mutually recursive STM's, then, we can simply write programs that differ only in the first bit of this data. Each of the two machines can do its own thing, according to the value of this bit; and each can also easily convert its own, directly available, program code to the other's, for the sake of mutual recursion.

To illustrate the utility of our newly enhanced STM model in the simplest possible setting, we give a transparent alternative to the usual obscure proof of the "fixed-point" version of Kleene's recursion theorem [23]. The result itself will turn out to be machine-independent.

Recursion Theorem. For each total, computable program transformation $\tau$ : $\{0,1\}^{+} \rightarrow\{0,1\}^{+}$, there is a fixed point $e$, itself computable from a program code for $\tau$, such that $\varphi_{e}=\varphi_{\tau(e)}$.

Proof: For the desired fixed point, just take a code $e_{0}$ for a program that retrieves its own code $\left(e_{0}\right)$, transforms it (to $\left.\tau\left(e_{0}\right)\right)$, and then simulates the resulting machine $\left(M_{\tau\left(e_{0}\right)}\right)$ on the given input.

One last useful tool lets us transform programs into "equivalent" ones that are longer. Codes $e_{1}$ and $e_{2}$ are equivalent if their STM's are equivalent (i.e., if $\varphi_{e_{1}}=\varphi_{e_{2}}$ and $S_{e_{1}}=S_{e_{2}}$ ). 
Padding Lemma. There is a one-to-one, computable "padding" transformation $p:\{0,1\}^{+} \rightarrow\{0,1\}^{+}$such that, for each $S T M$ code $e, p(e)$ is a longer code equivalent to $e$. Moreover, the corresponding unpadding transformation, that transforms $e$ to the shortest code $u(e)$ from which $e$ can be obtained by repeated application of $p$, is so simple that an STM with access to e can simulate access to $u(e)$ at no additional cost in space.

Proof: If $e$ includes no instructions to retrieve its own code, and if its "data segment" contains only 0 's, then just add one more 0 to the data. Otherwise, straightforwardly transform, in some standard, simple, one-to-one way, to a code that does not include self-retrieval instructions, and that has an empty data segment.

Proof of the compression theorem: Any constructible $S$ will do. There is a constructible space bound above any computable function, since an STM can compute the function and then go on to extend its storage tape according to the computed value.

We define $P(x)$ for one $x$ at a time, considering input strings $x$ in their natural order $<$. (The natural order is by length, with strings of the same length ordered lexicographically.) To keep $P$ hard enough, we aim to build in an exception to $P=\varphi_{e}$ whenever $S_{e} \nsucceq S$, by "cancelling" $e$ on some input $x$. To cancel $e$ on input $x$, we aim simply to choose $P(x)$ to be the least member of $\{0,1\}$ not equal to $\varphi_{e}(x)$. On input $x$, we aim to cancel the least $e$ (in that same natural order of binary strings) that should be, but has not yet been, cancelled.

Naively, we might try, on input $x$, to cancel $c(x)=\min \left\{e \mid S_{e} \nsucceq S\right.$ and $e$ is not $c\left(x^{\prime}\right)$ for any earlier $\left.x^{\prime}\right\}$. Since $P$ has to be computable and not too complex, however, this does not work. To salvage the plan, we must exploit its slack

1. There is no harm in cancelling a few extra e's, perhaps based on mere "suspicion" that $S_{e} \nsucceq S$

2. There is no harm in cancelling some e's more than once, perhaps saving much of the space necessary for a full check into past accomplishments.

3. There is no harm in cancelling some e's earlier or later than we had planned, perhaps saving much of the space necessary to determine exactly the originally intended ordering.

4. There is no harm in never explicitly cancelling some of those e's for which $\varphi_{e}$ is not total, since $P$ will differ from these merely by being total.

Taking advantage of all these observations, we decide, on input $x$, to cancel

$c(x)=\min \left\{e \mid S(x)\right.$ is enough space to simulate $M_{e}$ on input $x$,

but not enough to discover that $e=c\left(x^{\prime}\right)$ for some $\left.x^{\prime}<x\right\}$

(When this set is empty, arbitrarily cancel some fixed $e$ with $S_{e}$ identically equal to 1.) Note that it is the first condition that raises suspicion that maybe $S_{e} \nsucceq S$. The second condition might be satisfied either because space $S(x)$ is inadequate for the review, or simply because $e$ has not yet been cancelled; our choice of $c(x)$ is based on the latter hope.

The key to the tightness of our compression theorem is that a single "universal" STM, given an arbitrary STM program code $e$ on its storage tape, can simulate $M_{e}$ in space equal to just $S_{e}(x)$ plus some constant $c_{e}$ that depends only on $e$. Assuming an appropriate (but straightforward) formalization and encoding, in fact, $c_{e}=|e|$ will suffice; the idea is for the universal STM to maintain on its storage tape an upto-date replica of $M_{e}$ 's storage tape, but with a copy of a slightly modified version of the self-delimiting program code $e$ inserted at the current head position. The main reason for modification of the code is to indicate which is the current control state of $M_{e}$; redundant, and hence temporarily changeable, copies of each bit facilitate 
such marking. Such marking also makes it possible to compare state labels, in order to find each next control state of $M_{e}$

In terms of the simulation we have outlined, the condition that " $S(x)$ is enough space to simulate $M_{e}$ on input $x$ " becomes simply " $S(x) \geq S_{e}(x)+|e|$ ". Once $e=$ $c(x)$ is known, therefore, it will be straightforward by simulation within space $S(x)$ to compute $\varphi_{e}(x)$ for the sake of diagonalization.

To compute $c(x)$ within space $S(x)$, we use a recursive procedure. The first step is to lay out space exactly $S(x)$. Within that space, never again extending the storage tape, we write down each successive binary string $e$ until we find one that qualifies to be $c(x)$, or until the next one would not fit within the allocated space. For each such $e$, we check whether universal simulation of $M_{e}$ on input $x$ overflows the allocated space. (Except when the simulation fills the entire tape, this requires recognition of a simulated right end that is not the actual right end of the storage tape. A suitable convention under such circumstances is to maintain a 1 in the first unused position, and 0 's in all the rest.)

If the universal simulation does overflow, then $e$ does not satisfy the condition $S(x) \geq S_{e}(x)+|e|$, and we go on to the next value of $e$. If it does not overflow, then we go on to check the second condition, leaving the self-delimited string $e$ written at the beginning of the storage tape. Within the remaining space $S(x)-|e|$, we write down a self-delimited version of each successive binary string $x^{\prime}$ that precedes $x$, until we find one with $c\left(x^{\prime}\right)=e$, or until the next $x^{\prime}$ would not fit within the allocated space. To check each $x^{\prime}$, we try recursively to calculate $c\left(x^{\prime}\right)$ within the remaining available space. This involves retrieval of the code $d$ for the STM we are designing and universal simulation of $M_{d}$ on $x^{\prime}$; it is easy for the simulation to recognize $e$ and the representation of $x^{\prime}$ as a bound, because they are both selfdelimiting. If we run out of space before finding $c\left(x^{\prime}\right)$, or if $c\left(x^{\prime}\right) \neq e$, then we go on to the next value of $x^{\prime}$; but, if we discover that $c\left(x^{\prime}\right)=e$, then $e$ does not satisfy the second condition, and we go on to its next value.

Having thus shown that an STM can compute $P$ within space $S$, we turn to the converse, that $M_{e}$ cannot compute $P$ if $S_{e} \nsucceq S$. We have noted already that $M_{e}$ cannot compute $P$ unless $\varphi_{e}$ is total; and, by explicit design, $M_{e}$ does not compute $P$ if $e$ gets cancelled. Therefore, it suffices to show that $e$ gets cancelled if $\varphi_{e}$ is total and $S_{e} \nsucceq S$. Noting that the latter condition implies that both $S(x)-S_{e}(x)$ and $S(x)$ itself are simultaneously unbounded, just consider the first $x$ so large that every $e^{\prime}<e$ that ever gets cancelled is $c\left(x^{\prime}\right)$ for some $x^{\prime}<x$, and such that $S(x)$ is enough space both to simulate $M_{e}$ on input $x\left(S(x)-S_{e}(x) \geq|e|\right)$ and to discover an earlier cancellation of each such $e^{\prime}$. For that $x, e=c(x)$.

Before leaving the compression phenomenon, we should note that restricting space can have a drastic effect even on predicates that do remain computable. The effect is in terms of minimum program size. If we define

$$
K(P, S)=\min \left\{|e| \mid M_{e} \text { computes predicate } P \text { within space } S\right\},
$$

for example, then we can prove the following abrupt trade-off between program size and space complexity [17]:

Theorem. Above any computable function, and for any "limit-computable" function $h: N \rightarrow N$ (" $h$ " for "huge"), there is a computable space bound $S: \Sigma^{+} \rightarrow N$ for which there is a predicate $P: \Sigma^{+} \rightarrow\{0,1\}$, computable within every space bound (even a constant one), but such that $K\left(P, S^{\prime}\right)>h\left(K(P, S)\right.$ ) unless $S^{\prime}(x) \succeq S(x)$.

( $h$ is defined to be limit-computable if $h(n)=\lim _{m \rightarrow \infty} g(m, n)$ for each $n$, for some computable function $g$.)

Proof idea: Adapt the proof of the compression theorem by adding the following additional requirement for membership of $e$ in the set of candidates for $c(x)$ : 
It "looks like" $|e|$ does not exceed $h\left(\left|e_{0}\right|\right)$, where $e_{0}$ is the program code for the STM being designed.

To implement the "looks like" aspect, make use of the approximation $g\left(m,\left|e_{0}\right|\right)$, for $m$ as large as possible within the available space. If $S(x)$ tends to infinity, then the set will be empty when $x$ gets sufficiently large, so that we are free to make $P(x)=0$ almost everywhere (i.e., with only finitely many exceptions).

Through mutual recursion, one can work out multistep versions of the above trade-off. See [24] for the details, and for many more results on trade-offs between program size and computational complexity, in some very general settings.

\section{Gaps and Arbitrary Speedup}

Before we turn explicitly to the speedup phenomenon, let us reexamine the specification of the predicate $P$ in the proof of the compression theorem. The mere definition of the cancellation function, and hence of $P$ itself, does not depend on the constructibility of $S$. If $S$ is merely computable, we still get a computable predicate $P$, that is not computable within space $S_{e}$ unless $S_{e} \succeq S$. On the other hand, for $P$ to be computable within space $S_{e} \geq S$, it suffices to be able to delimit space $S(x)$ within already constructed, or "preallocated", space $S_{e}(x)$. If computable $S$ is delimitable in this sense within every $S_{e} \geq S$, then let us call $S$ subconstructible. From each subconstructible $S$, then, our specification gives us a predicate $P$, that is computable within space $S_{e}$ if and only if $S_{e} \succeq S$.

Lemma 1. If $S: \Sigma^{+} \rightarrow N$ is subconstructible, then there is a predicate $P$ : $\Sigma^{+} \rightarrow\{0,1\}$, computable within space $S_{e}$ if and only if $S_{e} \succeq S$.

We will see below that this slight generalization of the compression theorem is a major step in the proof of the speedup theorem.

Remark. It follows from the Fundamental Theorem [11, 12, 19] (discussed below) that the subconstructibility hypothesis in Lemma 1 can be weakened all the way to mere computability. For the compression theorem, on the other hand, it is not enough for $S$ to be computable. The disparity is explained by the "gap" phenomenon.

The weak version of the gap phenomenon is that there are computable space bounds $S: \Sigma^{+} \rightarrow N$ such that whatever an STM can do within space $S$, it can actually do in much less space, so that there is a "complexity gap" below $S$, in which no function's inherent space complexity lies. In fact it is easy to design $S$ with the even stronger property that not even one particular STM can use space that lies in the gap infinitely often $[28,6]$; thus there are computable space bounds with no constructible space bounds anywhere nearby, explaining the disparity described above. For a mundane "doubly exponential" gap, for example, one can design $S$ as follows: For each $x$, choose $S(x)$ so that the interval from $S(x)$ down to $\log \log S(x)$ misses $S_{e}(x)$ for every $e \leq x$. It is straightforward to check whether a prospective choice is satisfactory; and there are only a finite number of spoiling values $S_{e}(x)$ to "dodge". With more effort, one can obtain a slightly (but necessarily) weaker gap as big as any "computable operator that preserves totalness" $[8,30]$. We state this result below, without proof.

A computable operator is a mapping from computable partial functions to computable partial functions, that can be specified by a computable program transformation. More formally, using STM codes as our programs, $F$ is a computable 
operator if and only if there is a computable transformation $\tau:\{0,1\}^{+} \rightarrow\{0,1\}^{+}$ for which $F\left(\varphi_{e}\right)=\varphi_{r(e)}$. (Note that not every computable transformation $\tau$ specifies an operator: We must have $\varphi_{\tau(e)}=\varphi_{\tau\left(e^{\prime}\right)}$ whenever $\varphi_{e}=\varphi_{e^{\prime}}$.) If the image of each total function is total, then we say that $F$ preserves totalness.

Gap Theorem for STM space. For each computable operator $F$ that preserves totalness, there are computable space bounds $S: \Sigma^{+} \rightarrow N$ and $S^{\prime} \leq S$ so much smaller that $F\left(S^{\prime}\right) \leq S$, but such that, for each $e$, if $S_{e} \leq S$ holds almost everywhere, then so does $S_{e} \leq S^{\prime}$.

Finally, we state our speedup theorem, also in terms of computable operators [18].

"Speedup" Theorem for STM space. For each computable operator $F$ that preserves totalness, there is a computable predicate $P: \Sigma^{+} \rightarrow\{0,1\}$ with " $F$-speedup": Whenever $P$ is computable within a space bound $S$, it is also computable within a space bound $S^{\prime} \leq S$ so much smaller that $F\left(S^{\prime}\right) \leq S$ holds almost everywhere.

By induction, of course, the speedup theorem will yield an entire infinite, rapidly descending sequence $S, S^{\prime}, S^{\prime \prime}, \ldots$ Note that the finitely many exceptions to each "descent" $F\left(S^{(i+1)}\right) \leq S^{(i)}$ cannot be avoided: Even if $F(f)$ were just $f+1$, for each particular argument $x$ we would reach a contradiction to perfect speedup after just $S(x)$ successive applications. On the other hand, one major step in the proof is showing that, with only finitely many exceptions at each step, such rapid descent is possible.

Lemma 2. For each computable operator $F$ that preserves totalness, there is a sequence of functions $g_{1} \geq g_{2} \geq g_{3} \geq \cdots\left(g_{i}: \Sigma^{+} \rightarrow N\right)$ such that, for each $i$, $F\left(g_{i+1}\right) \leq g_{i}$ holds almost everywhere. Moreover, the sequence $\left\{g_{i}\right\}$ is uniformly computable, and even "uniformly subconstructible", as defined below.

A sequence $\left\{g_{i}\right\}$ is uniformly subconstructible if some single STM, given $x$ on its input tape and $i$ on its otherwise blank preallocated storage tape of length $m \geq|i|$, can halt with the storage tape head at position $\min \left\{m, g_{i}(x)\right\}$ and with no net change to the content of the storage tape. The need for some such condition is dictated by Lemma 3 , the one missing link to complete a proof of the speedup theorem based on Lemmas 1 and 2 .

Lemma 3. For each uniformly subconstructible decreasing sequence $g_{1} \geq g_{2} \geq g_{3} \geq$ $\cdots\left(g_{i}: \Sigma^{+} \rightarrow N\right)$, there is a single subconstructible space bound $S: \Sigma^{+} \rightarrow N$ such that each $e$ satisfies $S_{e} \succeq S$ if and only if it satisfies $S_{e} \succeq g_{i}$ for some $i$.

It remains only to prove Lemmas 2 and 3 . Since the demands of the latter dictate the appropriate notion of uniform subconstructibility, we give its proof first.

Proof of Lemma 3: The argument is reminiscent of the proof of the compression theorem. We define $S(x)$ for one $x$ at a time, again considering input strings $x$ in their natural order. Again the specification involves diagonalization, but the task seems more difficult this time: If there is no $i$ for which $S_{e} \succ g_{i}$, then we must design $S$ so that $S_{e} \nsucceq S$; but setting no single value of $S$ can guarantee this, thereby decisively "cancelling" $e$. All we can do on a particular input $x$ is to "attack" some $e=a(x)$. The problem turns out not to be a real one, however, since, by the padding lemma, there are so many other STM codes $e^{\prime}$ with $S_{e^{\prime}}$ identical to $S_{e}$.

To attack $e$ on input $x$, we simply define $S(x)$ to be $g_{e}(x)$ (identifying $e$ with its position in the standard ordering of binary strings). On the assumption that there is no $i$ for which $S_{e} \succeq g_{i}$, we have in particular that $S_{e} \nsucceq g_{e}$, so that the choice of $S(x)$ is a modest step toward arranging $S_{e} \nsucceq S$. In terms of the "attack function" $a$, our definition is simply

$$
S(x)=g_{a(x)}(x)
$$


to complete the definition of $S$, it remains only to define $a$.

On input $x$, we attack STM code

$$
\begin{aligned}
& a(x)=\min \left\{e \mid g_{e}(x) \text { is enough space to simulate } M_{e} \text { on input } x,\right. \\
& \text { but not enough to discover that } \left.e=a\left(x^{\prime}\right) \text { for some } x^{\prime}<x\right\} .
\end{aligned}
$$

(When this set is empty, we conservatively take $a(x)=1$ and attack the very first STM code, thus defining $S(x)$ to be $g_{1}(x)$.) Note that the first condition raises suspicion that $S_{e} \nsucceq g_{e}$, and hence at least a faint suspicion that there is no $i$ for which $S_{e} \succeq g_{i}$.

Based only on some fixed algorithm for computing $g_{i}(x)$ from $i$ and $x$, we can implement the recursive review as in the proof of the compression theorem, to get a total attack function $a(x)$ and a resulting computable space bound $S(x)$. It remains only to show, for each $e$, that $S$ satisfies $S \preceq S_{e}$ if and only if some $g_{i}$ satisfies $g_{i} \preceq S_{e}$, and to show that $S$ is subconstructible.

First, note that $e$ does get attacked (i.e., $e=a(x)$ for some $x$ ) if it satisfies $S_{e} \nsucceq g_{e}$. The argument is familiar from the proof of the compression theorem: Just consider the first $x$ so large that every $e^{\prime}<e$ that ever gets attacked is $a\left(x^{\prime}\right)$ for some $x^{\prime}<x$, and such that $g_{e}(x)$ is enough space both to simulate $M_{e}$ on input $x$ $\left(g_{e}(x)-S_{e}(x) \geq|e|\right)$ and to discover an earlier attack on each such $e^{\prime}$. For that $x$, $e=a(x)$.

By the padding lemma, there is an infinite sequence of STM codes $e_{1}<e_{2}<$ $e_{3}<\cdots$ such that $S_{e}=S_{e_{1}}=S_{e_{2}}=S_{e_{3}}=\cdots$. If there is no $i$ for which $g_{e_{i}} \preceq S_{e}$, then it follows that $S_{e_{i}} \nsucceq g_{e_{i}}$ holds for every $i$, so that each $e_{i}$ gets attacked. If $e_{i}=a(x)$, then $S(x)=g_{e_{i}}(x) \geq S_{e}(x)+\left|e_{i}\right|$. Therefore, $S(x)-S_{e}(x)$ gets arbitrarily large, so that $S \npreceq S_{e}$.

Conversely, suppose $g_{i} \preceq S_{e}$. To show that $S \preceq S_{e}$, it suffices to show that $S(x)$ is bounded by $g_{i}(x)$ whenever both $x$ and $S(x)$ are sufficiently large. We do this by showing that $a(x)>i$ holds under the latter circumstances, so that $S(x)=g_{a(x)}(x) \leq g_{i}(x)$.

The argument that $a(x)$ exceeds $i$ whenever $x$ and $S(x)$ are sufficiently large is again familiar. Just consider $x$ so large that every $e^{\prime} \leq i$ that ever gets attacked is $a\left(x^{\prime}\right)$ for some $x^{\prime}<x$, and $S(x)$ so large that it is enough space to discover the earliest attack on each such $e^{\prime}$. Then, since $g_{a(x)}(x)=S(x), a(x) \leq i$ would imply choice of $a(x)$ in violation of the second requirement.

Finally, we must show how to "subconstruct" $S$. The idea is to try, within the preallocated space, for each successive STM code $e$, to lay out space $g_{e}(x)$ and to check within that space whether $e$ satisfies the two qualification conditions for $a(x)$. (It is the successive subconstructions that dictate the need for uniform subconstructibility. Note the importance of not losing track of $e$ in the subconstruction of $g_{e}(x)$, since the process must be able to continue later with the correct next value.) It might be necessary to skip the first few values of $e$, since, for those values, $g_{e}(x)$ might exceed the preallocated space; but, if one of these skipped values is $a(x)$, then $S(x)=g_{a(x)}(x)$ exceeds the preallocated space, so that subconstructibility places no constraint at all on the outcome. So when the first $e$ qualifies, we can assume that $a(x)$ is that $e$. Similarly, we can assume $a(x)=1$ if we discover that no $e$ will qualify. It is straightforward in either case to subconstruct space $g_{a(x)}(x)=S(x)$, preallocation permitting.

Proof of Lemma 2: The obvious idea is to design a recursive STM to uniformly subconstruct an appropriate sequence $g_{1} \geq g_{2} \geq g_{3} \geq \cdots$. Given inputs $i$ and $x$, and a space preallocation, the machine should try to calculate $F\left(g_{i+1}\right)(x)$ in unary within the preallocated space, saving $i$ and subconstructing the "high-water mark", the rightmost position needed for the calculation. This will guarantee that $g_{i} \geq$ 
$F\left(g_{i+1}\right)$ (everywhere!), but it will not guarantee that the $g_{i}$ 's are total. (We have already observed that they cannot all be total if there are no exceptions to the inequalities $g_{i} \geq f\left(g_{i+1}\right)$.)

Because $F$ preserves totalness, we do know that each $g_{i}$ will be total if $g_{i+1}$ is. We modify our design to include one auxiliary partial function $g_{0}$ such that every other $g_{i}$ is total if $g_{0}$ is not total, and also such that $g_{0}$ is total if and only if every other $g_{i}$ is total, all while introducing only finitely many exceptions to each inequality $g_{i} \geq F\left(g_{i+1}\right)(i \geq 1)$ : As before, given inputs $i$ and $x$, and a space preallocation, the machine works within the preallocated space, saving $i$ and finally subconstructing the high-water mark. If $i=0$, it tries to calculate $g_{j}(y)$ in unary for all $j, y<x(j \neq 0)$, finally concluding its subconstruction task by restoring the contents of the storage tape and positioning the storage head at the high-water mark. (This guarantees that $g_{0}$ will be total if and only if $g_{j}$ is total for every $j>0$.) If $i>0$, it first tries for $|x|$ steps to calculate $g_{0}(z)$ for all $z<i$. If it fails to complete the calculation of any of these values (for lack of either space or time), then it concludes its subconstruction at this point. (If $g_{0}$ is not total, it will fail whenever $i$ is sufficiently large, thus guaranteeing that $g_{i}$ is total for each such $i$. Since we will continue to have $g_{i}(i \geq 1)$ total whenever $g_{i+1}$ is, this will guarantee, in turn, that $g_{i}$ is total for every $i \geq 1$.) On the other hand, if it successfully calculates $g_{0}(z)$ for every $z<i$, as it is in fact bound to do for every sufficiently large preallocation and input string $x$, then it goes on with the original plan, trying to calculate $F\left(g_{i+1}\right)(x)$ in unary before concluding the subconstruction of the high-water mark.

\section{Effective Speedup}

We say that $f$ has effective $F$-speedup if there is a computable (possibly partial) program transformation $\sigma$ such that, if $M_{e}$ computes $f$, then $(\sigma(e)$ is defined and) so does $M_{\sigma(e)}$, with $F\left(S_{\sigma(e)}\right) \leq S_{e}$ almost everywhere. The additive-constant speedup of our first proposition, for example, is effective for every $f$. (The implicit operator there maps $S$ to $S^{\prime}$, where $S^{\prime}(x)=1$ if $S(x)=1$, and $S^{\prime}(x)=S(x)+1$ otherwise.) The arbitrarily dramatic speedup of our speedup theorem, on the other hand, cannot be effective.

Theorem (noneffectiveness of speedup). For no sufficiently large computable operator $F$ that preserves totalness is there a computable function with effective $F$-speedup.

Proof [5]: Suppose $F$ is large (enough so that the difference $F(S)-S$ is superlinear, say) and $f$ has effective $F$-speedup via $\sigma$. Fix some STM $M$ that computes $f$, say within space $S$ (necessarily large, of course). Without loss of generality, we can assume that $\sigma$ is total, and that $S_{\sigma(e)} \leq S$ for every e. (If not, then transform instead to $\sigma^{\prime}(e)$ such that, on input $x, M_{\sigma^{\prime}(e)}$ first tries, within relatively insignificant space $|x|$, say, to calculate $\sigma(e)$. If $M_{\sigma^{\prime}(e)}$ does not find $\sigma(e)$, then it goes on to behave just like $M$. If it does find $\sigma(e)$, then it behaves, by alternating simulation, like whichever of $M$ and $M_{\sigma(e)}$ requires less space for simulation on input $x$. (The respective requirements are at most $S(x)$ and $S_{\sigma(e)}(x)+|\sigma(e)|$.) The result is that $S_{\sigma^{\prime}(e)} \leq S$ for every $e$, and that $f$ has $F^{\prime}$-speedup via $\sigma^{\prime}$, for $F^{\prime}$ only slightly smaller than $F$.)

The strategy is somehow to design an STM $M_{e}$ that computes $f$ by computing and simulating its own speedup $M_{\sigma(e)}$. Such an $M_{e}$ would compute $f$ nearly as efficiently as $M_{\sigma(e)}$, a contradiction. 
It is easy to design $M_{e}$ to compute $f$, and it is easy to design $M_{e}$ to simulate $M_{\sigma(e)}$, but it seems hard to design $M_{e}$ to do both. The trick is to design two STM's, $M_{e_{0}}$ and $M_{e_{1}}$, interdependent through mutual recursion. On a particular input, one of the two could make sure to compute $f$, and the other could behave like $M_{\sigma\left(e_{0}\right)}$ or $M_{\sigma\left(e_{1}\right)}$, whichever requires less space. If we assign the two roles appropriately, then the machine not explicitly computing $f$ will be behaving like the (would-be) speedup of the other, and hence hopefully computing $f$ for that reason. Because our tentative argument is circular, however, with $\varphi_{e_{0}}=\varphi_{e_{1}}=f$ and $\varphi_{e_{i}}=\varphi_{\sigma\left(e_{i}\right)}$ depending on each other, we need an additional delicate "recursive review" patch.

On input $x$, within (relatively insignificant) space $|x|$, for as long an initial segment of inputs $x^{\prime}<x$ as possible, $M_{e_{i}}$ checks the three-way equality $\varphi_{\sigma\left(e_{0}\right)}\left(x^{\prime}\right)=$ $\varphi_{\sigma\left(e_{1}\right)}\left(x^{\prime}\right)=f\left(x^{\prime}\right)$ and whether or not $x^{\prime}$ is "special". It declares $x$ itself to be special if it is the very first input or if all the space allocated was needed to reach the last successfully checked special $x^{\prime}$. (By induction, $x$ will be the very next special input after $x^{\prime}$ in the latter case, and the sequence of special inputs will be infinite.) If $x$ is not special, or if the three-way equalities did not all hold, then $M_{e_{i}}$ just goes on to behave like $M$ on $x$, explicitly computing $f(x)$. Otherwise (i.e., if $x$ is special and all the successfully checked three-way equalities did hold), $M_{e_{1}}$ determines by alternating simulation which of $M_{\sigma\left(e_{0}\right)}$ and $M_{\sigma\left(e_{1}\right)}$ requires less space on the current input, $x$. (Arbitrarily break ties in favor of $M_{\sigma\left(e_{0}\right)}$, say.) If $M_{\sigma\left(e_{i}\right)}$ requires less space, then $M_{e_{i}}$ again goes on to behave like $M$ on $x$. Otherwise, $M_{e_{i}}$ goes on to behave like $M_{\sigma\left(e_{1-i}\right)}$ on $\boldsymbol{x}$.

Claim. Both $M_{e_{0}}$ and $M_{e_{1}}$ do compute $f$.

Proof: If not, then consider the least $x$ for which $\varphi_{e_{0}}(x)$ or $\varphi_{e_{1}}(x)$ differs from $f(x)$. The two cases are symmetric, so assume it is $\varphi_{e_{0}}(x)$ that differs from $f(x)$. By design, $x$ must be special, and we must have $\varphi_{e_{0}}(x)=\varphi_{\sigma\left(e_{1}\right)}(x)$ and $\varphi_{e_{1}}(x)=f(x)$, with $\varphi_{\sigma\left(e_{1}\right)}(x) \neq f(x)$. For each $x^{\prime}<x, \varphi_{e_{1}}\left(x^{\prime}\right)=f\left(x^{\prime}\right)$ by assumption; and, for each $x^{\prime}>x$, we will have $\varphi_{e_{1}}\left(x^{\prime}\right)=f\left(x^{\prime}\right)$ by design. (On each special $x^{\prime}>x$, $M_{e_{1}}$ will discover that $\varphi_{\sigma\left(e_{1}\right)}(x) \neq f(x)$.) Therefore, $\varphi_{e_{1}}$ is $f$ but $\varphi_{\sigma\left(e_{1}\right)}$ is not, a contradiction.

It follows that all the three-way equalities will always check out. Either for $i=0$ or for $i=1$, therefore, there will be an infinite sequence of special inputs $x$ on which $M_{e_{i}}$ ends up behaving like $M_{\sigma\left(e_{1-i}\right)}$ on $x$ and using space little more than $S_{\sigma\left(e_{1-i}\right)}(x) \leq S_{\sigma\left(e_{i}\right)}(x)$. Since $F$ is large, this contradicts the original supposition that $F\left(S_{\sigma\left(e_{i}\right)}\right) \leq S_{e_{i}}$ holds almost everywhere.

Effective speedup of a weaker sort is possible. It is possible, for example, if we weaken our requirement from " $F\left(S^{\prime}\right) \leq S$ almost everywhere" to just " $F\left(S^{\prime}\right) \leq S$ infinitely often (i.o.)" (i.e., $F\left(S^{\prime}\right)(x) \leq S(x)$ for infinitely many values of $x$ ). In fact, it is easy to arrange such "i.o.-speedup" all the way down to a trivial complexity level:

"Leveling" Theorem for STM space. For each computable space bound $S$ : $\Sigma^{+} \rightarrow N$, there is a computable predicate $P: \Sigma^{+} \rightarrow\{0,1\}$, not computable within space $S$, but with "i.o.-speedup to triviality", in the following sense: Whenever $P$ is computable within a space bound $S^{\prime}$, it is also computable within a space bound $S^{\prime \prime} \leq S^{\prime}$ so often so much smaller that the following set is infinite:

$$
\left\{x \mid S^{\prime}(x) \geq S(x) \text { and } S^{\prime \prime}(x)=1\right\} \text {. }
$$

Moreover, the speedup can be effective.

Proof [5]: We make $P$ different from every $\varphi_{e}$ with $S_{e} \leq S$ by straightforward diagonalization: For each $e$, if $S_{e}(e) \leq S(e)$, then we choose $P(e)$ to be the least 
member of $\{0,1\}$ not equal to $\varphi_{e}(e)$. (So $S_{e} \leq S \Rightarrow P(e) \neq \varphi_{e}(e) \Rightarrow P \neq \varphi_{e}$.) If $S_{e}(e)>S(e)$, then we choose $P(e)$ to be 0 , say. (Note, incidentally, that $P(x)$ is straightforwardly computable in just $|x|$ more space than $S(x)$ is.)

Now suppose $M_{e_{1}}$ computes $P$. Letting $p$ and $u$ be the padding and unpadding transformations discussed earlier, consider the input strings $u\left(e_{1}\right), p\left(u\left(e_{1}\right)\right)$, $p^{2}\left(u\left(e_{1}\right)\right), \ldots$ For each such string $e=p^{i}\left(u\left(e_{1}\right)\right), P(e)$ does equal $\varphi_{e_{1}}(e)$, so that we must have $S_{e}(e)>S(e)$ and $P(e)=0$. Because $e$ and $e_{1}$ are equivalent, we also have $S_{e_{1}}(e)>S(e)$. So, for the desired i.o.-speedup to triviality, design $M_{e_{2}}$ to output 0 without extending its storage tape if its input is a padded version of $u\left(e_{1}\right)$, and to behave exactly like $M_{e_{1}}$ otherwise.

\section{Fundamental Theorem for STM Space}

Before we go on to develop the notion of machine independence, and to derive machine-independent results from the machine-dependent ones we have proven so far, we should mention that our compression and speedup results for STM space complexity can be combined and strengthened to yield an exact characterization of those "prospective STM space complexities" that are "realizable".

A prospective (STM space) complexity is just a set $\mathcal{C}$ of STM codes. We write $\mathcal{C} \preceq S$ (or $S \succeq \mathcal{C}$ ) to indicate that $S_{e} \preceq S$ holds for some $e$ in $\mathcal{C}$. A computable function $f$ realizes prospective complexity $\mathcal{C}$ if, for each STM code $e, f$ is computable by an STM within space $S_{e}$ if and only if $S_{e} \succeq \mathcal{C}$.

We say that $\mathcal{C}$ and $\mathcal{C}^{\prime}$ are equivalent if, for each STM code $e, \mathcal{C} \preceq S_{e}$ holds if and only if $\mathcal{C}^{\prime} \preceq S_{e}$ does. Note that, if $f$ realizes $\mathcal{C}$, and if $\mathcal{C}^{\prime}$ is equivalent to $\mathcal{C}$, then $f$ realizes $\mathcal{C}^{\prime}$, too. It is easy to see that each realizable prospective complexity, or some equivalent one, must necessarily satisfy the following three conditions:

1. For some $e$ in $\mathcal{C}, S_{e}$ is total.

2. For each $e_{1}$ and $e_{2}$ in $\mathcal{C}$, there is some $e_{3}$ in $\mathcal{C}$ for which $S_{e_{3}} \preceq \min \left(S_{e_{1}}, S_{e_{2}}\right)$.

3. For some computable predicate $P(x, y, e), \mathcal{C}=\{e \mid \exists x \forall y P(x, y, e)\}$.

The characterization theorem [11,12,19], which Meyer and Winklmann call "The Fundamental Theorem of Complexity Theory", adds that these three conditions also suffice for realizability, even by a function that assumes only two values, so that they exactly characterize the realizable prospective STM space complexities:

Fundamental Theorem for STM space. The following are equivalent for a prospective complexity $\mathcal{C}$ :

(i) $\mathcal{C}$ is realizable.

(ii) $\mathcal{C}$ is realizable by a predicate.

(iii) $\mathcal{C}$ is equivalent to a prospective complexity that satisfies conditions 1-3 above.

To complete a proof of the Fundamental Theorem, it suffices to prove an alternative to our Lemma 2, saying that, for every prospective complexity $\mathcal{C}$ that satisfies the three conditions, there is a uniformly subconstructible decreasing sequence $\left\{g_{i}\right\}$, essentially as above, such that, for each STM code $e, S_{e} \succeq \mathcal{C}$ if and only if $S_{e} \succeq g_{i}$ for some $i$.

From the Fundamental Theorem, one can prove our versions of the compression and speedup theorems as easy corollaries. One can also conclude that, for every computable function, there is a predicate with exactly the same STM space complexity, strongly justifying the emphasis in complexity theory on computing predicates and recognizing languages. 


\section{Machine Independence}

Let us turn now to machine independence. A theorem on "machine-independent computational complexity" will have to be true for every "machine model" and "measure of computational complexity". To make this precise, we need rigorous but broad notions of what can be a machine model and what can be a complexity measure.

We consider the issue of machine model first. Recall that, in the case of the STM, we formalized and encoded machine descriptions to pin down the details of the model. The result was what we now call a programming system, in which each binary "program" code $e$ specifies a partial function $\phi_{e}$. Rather than generalize the informal notion of "machine model", we focus on the resulting programming systems, viewing each code $e$ as a "program" for, or particular instance of, some implicit "machine" that computes the partial function $\phi_{e}$.

Not every programming system is reasonable. One expectation by modern programmers and those familiar with the Church-Turing thesis [23] is that programs written in any feasible programming language can be translated, or "compiled", into any other serious programming language. Although there might be other reasonable expectations, this one weak requirement turns out to be enough for a useful definition [22]: A programming system $\left\{\phi_{c}\right\}$ is reasonable (or perhaps we should say "as reasonable as the STM system") if there exist computable translations (or "cross compilers") $f, g:\{0,1\}^{+} \rightarrow\{0,1\}^{+}$such that, for each program code $e, \phi_{e}=\varphi_{f(e)}$ and $\varphi_{e}=\phi_{g(e)}$. For any truly reasonable programming system, of course, the translations $f$ and $g$ would be efficiently computable; but, by adopting the more liberal definition, we will be able to see that the results we have been using and proving for STM's are machine-independent in the most robust sense.

For example, it already follows from our liberal definition that every reasonable programming system $\left\{\phi_{e}\right\}$ has all of the following properties [22, 15]:

1. (Computability) $\left\{\phi_{e}\right\}$ is the set of all computable partial functions.

2. (Uniform computability) $\phi_{e}(x)$ is a computable partial function of $e$ and $x$.

3. (Computable composition) There is a computable function $c$ such that, for each $e_{1}$ and $e_{2}, \phi_{c\left(e_{1}, e_{2}\right)}$ is the composition of $\phi_{e_{1}}$ and $\phi_{e_{2}}$ (i.e., $\phi_{c\left(e_{1}, e_{2}\right)}(x)=$ $\phi_{e_{1}}\left(\phi_{e_{2}}(x)\right)$ for every $\left.x\right)$.

4. (s-1-1 theorem) If we alternatively view each $\phi_{e}$ as a computable partial function of two variables, by some computable pairing convention, then there is a computable function $s$ such that, for each $e, x$, and $y, \phi_{s(e, x)}(y)=\phi_{e}(x, y)$.

5. (Recursion theorem) For each total, computable program transformation $\tau:\{0,1\}^{+} \rightarrow\{0,1\}^{+}$, there is a fixed point $e$, itself computable from a program code for $\tau$, such that $\phi_{e}=\phi_{\tau(e)}$.

6. (Padding lemma) There is a computable, one-to-one "padding" function $p$ of two variables such that $\phi_{e}=\phi_{p(e, i)}$ for every program code $e$ and integer $i$.

7. (Isomorphism theorem) There is a bijective (i.e., one-to-one and onto) computable translation $f$ such that $\phi_{e}=\varphi_{f(e)}$ for every program code $e$.

To see that every reasonable programming system $\left\{\phi_{e}\right\}$ has these seven properties, first note that the STM system does, and then try to translate the properties to $\left\{\phi_{e}\right\}$. For properties $1-4$, this is completely straightforward. To find the fixed point for property 5 , first find a fixed point $e^{\prime}$ for the composite transformation $f \circ \tau \circ g$ in the STM system, and then use its translation $g\left(e^{\prime}\right): \phi_{g\left(e^{\prime}\right)}=\varphi_{e^{\prime}}=$ $\varphi_{f\left(\tau\left(g\left(e^{\prime}\right)\right)\right)}=\phi_{\tau\left(g\left(e^{\prime}\right)\right)}$.

Assuming property 6, it is not hard to obtain the bijection for property 7 : Starting with an empty partial function $f$, extend both the domain and the range 
of $f$ to include each successive $e$. To assign $f(e)$ or $f^{-1}(e)$, translate $e$ and pad enough times to obtain an equivalent program that has not yet been assigned.

To prove property 6 , we would like to translate to the STM programming system, pad there, and translate back. There is a problem, however, because the translations might not be one-to-one. Property 7 would solve our problem, but we have already used property 6 in its proof. The solution is to settle first for a weakened version of property 7 , the proof of which is the only tricky step in the whole sequence.

Injection Lemma. Between any pair of reasonable programming systems, there is $a$ one-to-one computable translation.

Proof: Start with any computable translation $h$ from $\left\{\phi_{e}^{\prime}\right\}$ to $\left\{\phi_{e}\right\}$, and aim to replace the images $h(e)$ in the unprimed system by a sequence of respectively equivalent but distinct programs there.

In the unprimed programming system, let $s$ be the computable $s-1-1$ function of property 4 . We will choose $d_{0}$ so that, for each $e, s\left(d_{0}, e\right)$ will be usable as the desired replacement for $h(e)$.

We obtain $d_{0}$ as the fixed point of a computable program transformation $\tau$ that transforms program $d$ in such a way that

$$
\phi_{\tau(d)}(e, x)= \begin{cases}0 & \text { if } s(d, e) \in\left\{s\left(d, e^{\prime}\right) \mid e^{\prime}<e\right\} \\ 1 & \text { if not, but if } s(d, e) \in\left\{s\left(d, e^{\prime}\right) \mid e<e^{\prime} \leq x\right\} \\ \phi_{h(e)}(x) & \text { otherwise. }\end{cases}
$$

(To get such a transformation, straightforwardly design an STM to produce the desired output, and then translate the result into the unprimed abstract programming system.) Since $\phi_{s\left(d_{0}, e\right)}=\phi_{d_{0}}(e, x)=\phi_{\tau\left(d_{0}\right)}(e, x)$, this yields

$$
\phi_{s\left(d_{0}, e\right)}(x)= \begin{cases}0 & \text { if } s\left(d_{0}, e\right) \in\left\{s\left(d_{0}, e^{\prime}\right) \mid e^{\prime}<e\right\} \\ 1 & \text { if not, but if } s\left(d_{0}, e\right) \in\left\{s\left(d_{0}, e^{\prime}\right) \mid e<e^{\prime} \leq x\right\} \\ \phi_{h(e)}(x) & \text { otherwise. }\end{cases}
$$

To complete our proof, we must show that $s\left(d_{0}, e\right)$ is a one-to-one function of $e$, and that $\phi_{s\left(d_{0}, e\right)}=\phi_{h(e)}$ for every $e$. If the former is true, then certainly the latter will be true, since $s\left(d_{0}, e\right)$ will never belong to $\left\{s\left(d_{0}, e^{\prime}\right) \mid e^{\prime}<e\right\}$ or $\left\{s\left(d_{0}, e^{\prime}\right) \mid e<e^{\prime} \leq x\right\}$. Therefore, it remains only to show that $s\left(d_{0}, e\right)$ is one-toone.

For the sake of argument, suppose that $s\left(d_{0}, e\right)$ is not a one-to-one function of $e$. Let $s\left(d_{0}, e\right)=s\left(d_{0}, e^{\prime}\right)$ be a counterexample with $e<e^{\prime}$, and with $e$ as small as possible. Examining the cases, we see that $\phi_{s\left(d_{0}, e^{\prime}\right)}(x)=0$ for every $x$, and that $\phi_{s\left(d_{0}, e\right)}(x)=1$ for every $x \geq e^{\prime}$, contradicting $s\left(d_{0}, e\right)=s\left(d_{0}, e^{\prime}\right)$.

Our notion of a complexity measure on an arbitrary programming system $\left\{\phi_{e}\right\}$ should generalize our space measure $\left\{S_{e}\right\}$ on the STM programming system $\left\{\varphi_{e}\right\}$. To each partial function $\phi_{e}$, it should assign an appropriate "resource bound" $\Phi_{e}$. Since neither the "machine model" nor its "resource" is explicit, the constraints will have to be abstract axioms. In our liberal definition, there will be only two such axioms [3]:

A1. For each $e, \Phi_{e}$ is defined on precisely the same domain as $\phi_{e}$

A2. It is decidable from $e, x$, and $b$ whether $\Phi_{e}(x)=b$.

Note that the prototype measure $\left\{S_{e}\right\}$ on $\left\{\varphi_{e}\right\}$ does satisfy these axioms. The natural notions of time also satisfy them.

Our notion of complexity measure is so general that it actually obviates the need to look at more than one reasonable programming system $\left\{\phi_{e}\right\}$. If $\left\{\Phi_{e}^{\prime}\right\}$ is any 
complexity measure on any other reasonable programming system $\left\{\phi_{e}^{\prime}\right\}$, then there is a complexity measure $\left\{\Phi_{e}\right\}$ on $\left\{\phi_{e}\right\}$ that is equivalent in the following strong sense: For each $e$, there is an $e^{\prime}$ such that $\phi_{e^{\prime}}^{\prime}=\phi_{e}$ and $\Phi_{e^{\prime}}^{\prime}=\Phi_{e}$; and, for each $e^{\prime}$, there is an $e$ such that $\phi_{e}=\phi_{e^{\prime}}^{\prime}$ and $\Phi_{e}=\Phi_{e^{\prime}}^{\prime}$. In other words, each partial function is computable in exactly the same resource bounds in the two systems. Design of $\Phi_{e}$ is easy: Just take it to be $\Phi_{f(e)}^{\prime}$, where $f$ is a bijective computable translation from the unprimed programming system to the primed one. When it is convenient, therefore, we can restrict attention to any one particular programming system-to our formalization of STM's, for example. Of course complexity measures that seem natural in other programming systems might appear quite obscure when translated to STM's.

Through "cross compilation", it turns out that many complexity results carry over from one reasonable programming system and complexity measure to all others. This includes even the Fundamental Theorem, although the published versions $[14,25]$ are proved directly from the axioms. (In either case, the results are somewhat "blurred" and relatively awkward to state.) The key tool is the following consequence of our definitions:

Recursive-Relatedness Theorem [3]. If $\left\{\Phi_{e}\right\}$ and $\left\{\Phi_{e}^{\prime}\right\}$ are complexity measures on the respective reasonable programming systems $\left\{\phi_{e}\right\}$ and $\left\{\phi_{e}^{\prime}\right\}$, then there is a total, computable function $r$ such that, for each $e$, there is an $e^{\prime}$ such that $\phi_{e^{\prime}}^{\prime}=\phi_{e}$ and $\Phi_{e^{\prime}}^{\prime}={ }_{r} \Phi_{e}$, where the binary relation $=_{r}$ is the " $r$-blurred" version of equality defined below.

For $r: \Sigma^{+} \times N \rightarrow N$, the binary relation $=_{r}$ is defined in terms of the binary relation $\leq_{r}$. If $\psi_{1}$ and $\psi_{2}$ are partial functions from $\Sigma^{+}$to $N$, then we say that $\psi_{1} \leq_{r} \psi_{2}$ holds if $\psi_{1}(x) \leq r\left(x, \psi_{2}(x)\right)$ holds for almost every $x$, and we say that $\psi_{1}=_{r} \psi_{2}$ holds if both $\psi_{1} \leq_{r} \psi_{2}$ and $\psi_{2} \leq_{r} \psi_{1}$ hold. Note that, notation notwithstanding, neither $\leq_{r}$ nor $=_{r}$ is transitive, in general.

Remarks. (i) Machine-based complexity theory dwells on questions such as how small the function $r$ can be for specific programming systems and complexity measures of interest. (In general, of course, there might not be one best $r$; the recursive relation itself might happen to have speedup.) The issues include the relative efficiency of various machine models and the relationship between the time and space measures on any particular model.

(ii) Our development provides no good reason, except for technical need, to tolerate finitely many exceptions to the inequality defining $\leq_{r}$. In the concrete case of STM's, we can modify the behavior of any machine in an arbitrary way on any particular finite set of input strings, without affecting its behavior elsewhere; but this need not be the case in an abstract setting. Some have proposed the imposition of an additional "finite-patching" axiom, to better capture the notion of "naturalness" for a complexity measure [27].

Proof of the recursive-relatedness theorem: We can take $e^{\prime}$ to be $f(e)$ for any fixed computable translation $f$ from the unprimed programming system to the primed one. Certainly, then, $\phi_{e^{\prime}}^{\prime}=\phi_{e}$. If we take

$$
r_{1}(x, b)=\max \left\{\Phi_{f(e)}^{\prime}(x) \mid e \leq x \text { and } \Phi_{e}(x)=b\right\},
$$

then we get $\Phi_{f(e)}^{\prime}(x) \leq r_{1}\left(x, \Phi_{e}(x)\right)$ for every $x \geq e$, so that $\Phi_{f(e)}^{\prime} \leq_{r_{1}} \Phi_{e}$. (Note that all four of $\Phi_{e}, \phi_{e}, \phi_{f(e)}^{\prime}$, and $\Phi_{f(e)}^{\prime}$ share the same domain of definition. Therefore, the condition $\Phi_{e}(x)=b$ guarantees that $\Phi_{f(e)}^{\prime}(x)$ is defined and can be found by exhaustive search.) Similarly, if we take

$$
r_{2}(x, b)=\max \left\{\Phi_{e}(x) \mid e \leq x \text { and } \Phi_{f(e)}^{\prime}(x)=b\right\},
$$


then we get $\Phi_{e} \leq_{r_{2}} \Phi_{f(e)}^{\prime}$. If we define $r$ to be the pointwise maximum of $r_{1}$ and $r_{2}$, therefore, then we do get $\Phi_{f(e)}^{\prime}={ }_{r} \Phi_{e}$.

For Corollaries 1-4 below, let $\left\{\phi_{e}\right\}$ be any reasonable programming system, and let $\left\{\Phi_{e}\right\}$ be any complexity measure on $\left\{\phi_{e}\right\}$. For each resource bound $B: \Sigma^{+} \rightarrow N$, the complexity class defined or "named" by $B$, denoted here by

$$
\operatorname{CCLASS}_{\left\{\phi_{e}\right\},\left\{\Phi_{e}\right\}}(B)
$$

is the class of functions computable in a resource bound bounded by $B$ almost everywhere. We will omit the subscripts from the notation when they are clear from context (but not in Corollary 5, where they are not).

Corollary 1 ("blurred" compression). There is a computable "blurring" function $r$ such that, for each total resource bound $\Phi_{e}$, there is a predicate $P$ computable within some resource bound $\Phi_{e^{\prime}}={ }_{r} \Phi_{e}$, but not computable within a resource bound $\Phi_{e^{\prime \prime}}$ unless $\Phi_{e^{\prime \prime}} \geq_{r} \Phi_{e}$

Remarks. (i) Another issue in machine-based complexity is how tight the compression can be for a particular complexity measure on a particular programming system of interest. (Here, again, however, there might not be one best tightness $r$.)

(ii) There are similarly blurred (less abrupt) versions of the trade-offs between program size and computational complexity.

Proof of Corollary 1: Let $r_{0}$ recursively relate $\left\{\Phi_{e}\right\}$ on $\left\{\phi_{e}\right\}$ to $\left\{S_{e}\right\}$ on $\left\{\varphi_{e}\right\}$ where we already have a compression result, and take $r$ to be somewhat larger $\left(r(x, b)=\max \left\{r_{0}\left(x, b^{\prime}\right)\left|b^{\prime} \leq r_{0}(x, b)+\right| x \mid\right\}\right.$ will do.) If $\Phi_{e}$ is total, then recursive relatedness gives us a $d$ for which $S_{d}=r_{0} \Phi_{e}$. Compression for STM space gives us a predicate $P$ computable within space $S_{d^{\prime}}$ if and only if $S_{d^{\prime}} \succeq S_{d}$. We show that $P$ satisfies the conclusion of the asserted corollary.

Since $P$ is computable within space $S_{d}$, recursive relatedness guarantees that it is computable in the abstract system within some resource bound $\Phi_{e^{\prime}}=r_{r_{0}} S_{d}={ }_{r_{0}} \Phi_{e}$, which implies by composition that $\Phi_{e^{\prime}}={ }_{r} \Phi_{e}$. On the other hand, if $P$ is computable in the abstract system within resource bound $\Phi_{e^{\prime \prime}}$, then it is computable on an STM within a space bound $S_{d^{\prime \prime}}$ that must satisfy both $S_{d^{\prime \prime}}=r_{0} \Phi_{e^{\prime \prime}}$ (by recursive relatedness) and $S_{d^{\prime \prime}} \succeq S_{d}$ (by the inherent complexity of $P$ ). Therefore, $\Phi_{e^{\prime \prime}}=r_{0}$ $S_{d^{\prime \prime}} \succeq S_{d}=r_{0} \Phi_{e}$, so that $\Phi_{e^{\prime \prime}} \geq_{r} \Phi_{e}$.

Corollary 2. There is a total resource bound $\Phi_{e}$ above each computable function.

Corollary 3 (speedup). For each computable operator $F$ that preserves totalness, there is a computable predicate $P$ with " $F$-speedup": Whenever $P$ is computable within a resource bound $\Phi$, it is also computable within a resource bound $\Phi^{\prime}$ so small that $F\left(\Phi^{\prime}\right) \leq \Phi$ holds almost everywhere.

Corollary 4 (gap). For each computable operator $F$ that preserves totalness, there are computable resource bounds $\Phi$ and $\Phi^{\prime} \leq \Phi$ so much smaller that $F\left(\Phi^{\prime}\right) \leq \Phi$, but such that, for each $e$, if $\Phi_{e} \leq \Phi$ holds almost everywhere, then so does $\Phi_{e} \leq \Phi^{\prime}$.

Proof idea for Corollaries 2-4: As in the proof of Corollary 1, exploit the corresponding fact for STM space complexity. The "blurring" does not show up in any of the final results, because we can simply cite the STM result for a larger threshhold or operator than is our real goal.

Corollary 5. For any pair of complexity measures, on any pair of reasonable programming systems, there is a computable resource bound $B: \Sigma^{+} \rightarrow N$ that defines the same complexity class in both systems. (If the respective systems and complexity measures are given unprimed and primed versions of the usual names, then 
the conclusion is that there is always a bound $B$ for which $\operatorname{CCLASS}_{\left\{\phi_{e}\right\},\left\{\Phi_{e}\right\}}(B)=$ $\operatorname{CCLASS}_{\left\{\phi_{e}^{\prime}\right\},\left\{\Phi_{e}^{\prime}\right\}}(B)$.)

Proof idea: Take $B$ in the middle of a gap that is enough bigger than the recursive relationship between the two complexity measures.

Remark. Yet another pursuit of machine-based complexity is to show that particular complexity measures of interest can differ.

As an example of an interesting machine-independent theorem that apparently does not generalize by recursive relatedness, we give the "union theorem" [16]. The theorem states that the union of any uniform hierarchy of complexity classes is itself a complexity class. (Note that Lemma 3 above can be viewed as a sort of an intersection theorem. It follows from the speedup theorem, however, that the exact analogue cannot hold [1].)

Union Theorem. Consider any complexity measure on any reasonable programming system. For each uniformly computable nondecreasing sequence of resource bounds $B_{1} \leq B_{2} \leq B_{3} \leq \cdots\left(B_{i}: \Sigma^{+} \rightarrow N\right)$, there is a single computable resource bound $B_{\infty}: \bar{\Sigma}^{+} \rightarrow N$ for which

$$
\operatorname{CCLASS}\left(B_{\infty}\right)=\bigcup_{i=1}^{\infty} \operatorname{CCLASS}\left(B_{i}\right)
$$

Proof: What we really prove is even stronger, and has very little to do with complexity classes: Given any nondecreasing sequence of functions $\left\{B_{i}\right\}$ and any sequence of functions $\left\{\Phi_{e}\right\}$ to "dodge", there is a function $B_{\infty}$ that bounds every $B_{i}$ almost everywhere, and such that every $\Phi_{e}$ that is bounded by $B_{\infty}$ almost everywhere is already bounded by some $B_{i}$ almost everywhere. Moreover, $B_{\infty}$ is computable if $\left\{B_{i}\right\}$ and $\left\{\Phi_{e}\right\}$ are uniformly computable and $\left\{\Phi_{e}\right\}$ satisfies axiom A2 for complexity measures.

We define $B_{\infty}(x)$ for one $x$ at a time, considering arguments $x$ in their usual natural order. To insure that $B_{\infty}$ bounds every $B_{i}$ almost everywhere, we must take care that it should eventually stop dipping below each $B_{i}$; we will check this explicitly at the end of the proof. To dodge the functions we should dodge, we aim to "attack" each pair $(e, i)$ for which $B_{i}$ is not bounded almost everywhere by $\Phi_{e}$, and for which $i>e$ holds. An attack will consist of a distinct dip by $B_{\infty}$ below $\Phi_{e}$, so that, if $(e, i)$ gets attacked for all $i>e, B_{\infty}$ will dip below $\Phi_{e}$ infinitely often, as required.

Unsurprisingly, our strategy on argument $x$ is to attack the smallest pair $(e, i)$, if there is one, that satisfies $i<x$, that was not attacked on any earlier argument $x^{\prime}<x$, and that satisfies $\Phi_{e}(x)>B_{i}(x)$ (i.e., $\Phi_{e}(x)=b$ for no $b<B_{i}(x)$, which is decidable if axiom A2 holds). Since we attack no pair more than once, we do eventually attack every pair $(e, i)$ for which $\Phi_{e}>B_{i}$ holds infinitely often. The method of attack is simply to set $B_{\infty}(x)$ to $B_{i}(x)<\Phi_{e}(x)$. In the exceptional case that no pair is eligible for attack, set $B_{\infty}(x)$ to $B_{x}(x)$.

Finally, to see that our $B_{\infty}$ does eventually stop dipping below $B_{i}$, note that $B_{\infty}(x)<B_{i}(x)$ holds only if some pair $\left(e, i^{\prime}\right)$ with $e<i^{\prime}<i$ gets attacked on argument $x$, or if $x<i$. Since no pair is attacked twice, the number of such arguments $x$ is some finite number that depends only on $i$.

As a final example, we describe a version of the "honesty" or "naming" theorem of McCreight and Meyer [16]. Informally, the theorem states that every complexity class with a computable name also has an "honest" name, by which we mean a naming resource bound whose arguments and values are an "honest indication" of its complexity. More formally, call a function $B: \Sigma^{+} \rightarrow N$ h-honest if there is some 
program code $e$ for which $\phi_{e}$ is $B$, and for which $\Phi_{e}(x) \leq h(x, B(x))$ holds for almost every $x$. In the context of STM space complexity, for example, constructibility can be viewed as $h$-honesty for $h(x, B(x))=B(x)$. In this section, similarly, each total resource bound $\Phi_{e}$ is $h$-honest for this same $h$.

Honesty Theorem. For each computable resource bound $B: \Sigma^{+} \rightarrow N$, there is an $h$-honest resource bound $B^{\prime}: \Sigma^{+} \rightarrow N$ such that $\operatorname{CCLASS}\left(B^{\prime}\right)=\operatorname{CCLASS}(B)$, where $h: \Sigma^{+} \times N \rightarrow N$ is a computable function that depends only on the reasonable programming system and the complexity measure (and not on $B$ ).

Proof idea [20]: The construction, like the one for the union theorem, actually has very little to do with complexity classes. (In fact both results can be viewed as consequences of a single closure property for classes of program codes [9].) From $B=\phi_{e_{0}}$, we construct an honest bound $B^{\prime}$ in such a way that each $\Phi_{e}$ lies below $B^{\prime}$ almost everywhere if and only if it lies below $B$ almost everywhere.

For this construction, we do not define $B^{\prime}(x)$ strictly according to the natural order of its arguments $x$. Instead, we "dovetail" through an infinite number of "opportunities" for each of the infinitely many arguments $x$, until we discover the first "appropriate" opportunity to define each $B^{\prime}(x)$. If stage $n$ of the dovetailing is the $i$-th opportunity to define $B^{\prime}$ at argument $x$, then we consider defining $B^{\prime}(x)$ to be $i$.

The decision whether to define $B^{\prime}(x)=i$ at this stage $n$ is based on a growing, ordered agenda that we maintain. At stage $n$ there is one agenda item for each program code $e<n$. The agenda item for $e$ indicates whether or not we are looking currently for an(other) opportunity for $B^{\prime}$ to dip below $\Phi_{e}$. The decision is made as follows:

(1) Do not redefine $B^{\prime}(x)$ if it has been defined already at some stage $n^{\prime}<n$.

(2) Otherwise, do define $B^{\prime}(x)$ if the definition would fulfill a positive agenda item for some $e\left(i<\Phi_{e}(x)\right.$ for that $\left.e\right)$ without violating any negative item for an $e^{\prime}$ higher on the agenda than $e\left(i \geq \Phi_{e^{\prime}}(x)\right.$ for each such $\left.e^{\prime}\right)$.

The agenda is updated as follows:

(3) Add a negative item to the bottom of the agenda for program code $n$.

(4) If $\Phi_{e_{0}}(x)=i$, then convert to positive the agenda item for each $e$ such that $\Phi_{e}(x)>\phi_{e_{0}}(x)$. (The latter indicates a dip of $B=\phi_{e_{0}}$ below $\Phi_{e}$. The prerequisite $\Phi_{e_{0}}(x)=i$ insures that this dip is new.)

(5) If the decision was made in (2) to define $B^{\prime}(x)=i$, then convert to negative the highest positive responsible agenda item, and move that item to the bottom of the agenda.

For a careful proof that $B^{\prime}$ meets our specifications and is $h$-honest for some computable $h$ that depends only on $\left\{\phi_{e}\right\}$ and $\left\{\Phi_{e}\right\}$, see [20].

Finally, we should note that machine independence is known not to be generally decidable. In particular, it is not decidable from a property of computable partial functions whether the property is measure independent [2].

Acknowledgments. I thank George Hauser for his reactions to the earliest, least readable versions of this chapter; Jan van Leeuwen for his help and encouragement to finally finish; and Jun Tarui for constructive criticism of what I thought was the very last version. 
1. L. J. Bass, $A$ note on the intersection of complexity classes of functions, SIAM Journal on Computing 1, 4 (December 1972), 288-289.

2. V. L. Bennison, Recursively enumerable complexity sequences and measure independence, The Journal of Symbolic Logic 45, 3 (September 1980), 417-438.

3. M. Blum, A machine-independent theory of the complexity of recursive functions, Journal of the Association for Computing Machinery 14, 2 (April 1967), 322-336.

4. M. Blum, On the size of machines, Information and Control 11, 3 (September 1967), 257-265.

5. M. Blum, On effective procedures for speeding up algorithms, Journal of the Association for Computing Machinery 18, 2 (April 1971), 290-305.

6. A. Borodin, Computational complexity and the existence of complexity gaps, Journal of the Association for Computing Machinery 19, 1 (January 1972), 158-174.

7. C. Calude, Theories of computational complexity, Elsevier North-Holland, New York, 1988.

8. R. L. Constable, The operator gap, Journal of the Association for Computing Machinery 19, 1 (January 1972), 175-183.

9. P. van Emde Boas, Some applications of the McCreight-Meyer algorithm in abstract complexity theory, Theoretical Computer Science 7, 1 (August 1978), $79-98$.

10. J. Hartmanis and J. E. Hopcroft, An overview of the theory of computational complexity, Journal of the Association for Computing Machinery 18, 3 (July 1971), 444-475.

11. L. A. Levin, On storage capacity for algorithms, Soviet Mathematics Doklady 14, 5 (September-October 1973), 1464-1466.

12. L. A. Levin, Complexity of computation of computable functions (in Russian), in: Complexity of Computations and Algorithms, Kozmidiadi, Maslov, and Petri (eds.), "Mir", Moscow, 1974, pp. 174-185.

13. M. Li and P. M. B. Vitányi, Kolmogorov Complexity, this handbook.

14. N. Lynch, "Helping": Several Formalizations, The Journal of Symbolic Logic 40, 4 (December 1975), 555-566.

15. M. Machtey and P. Young, An introduction to the general theory of algorithms, Elsevier North-Holland, New York, 1978.

16. E. M. McCreight and A. R. Meyer, Classes of computable functions defined by bounds on computation: preliminary report, Conference Record of the ACM Symposium on Theory of Computing, Marina del Rey, California, May 1969, pp. 7988 .

17. A. R. Meyer, Program size in restricted programming languages, Information and Control 21, 4 (November 1972), 382-394.

18. A. R. Meyer and P. C. Fischer, Computational speed-up by effective operators, The Journal of Symbolic Logic 37, 1 (March 1972), 55-68. 
19. A. R. Meyer and K. Winklmann, The fundamental theorem of complexity theory (preliminary version), Foundations of Computer Science III, Part 1: Automata, Data Structures, Complexity, J. W. de Bakker and J. van Leeuwen (eds.), Mathematical Centre Tracts 108, Amsterdam, 1979, pp. 97-112.

20. R. Moll and A. R. Meyer, Honest bounds for complexity classes of recursive functions, The Journal of Symbolic Logic 39, 1 (March 1974), 127-138.

21. R. W. Ritchie, Classes of predictably computable functions, Transactions of the American Mathematical Society 106 (January 1963), 139-173.

22. H. Rogers, Jr., Gödel numberings of partial recursive functions, The Journal of Symbolic Logic 23, 3 (1958), 331-341.

23. H. Rogers, Jr., Theory of recursive functions and effective computability, McGraw-Hill, New York, 1967.

24. J. S. Royer and J. Case, Progressions of relatively succinct programs in subrecursive hierarchies, Technical Report 86-007, University of Chicago, Department of Computer Science, October 1986.

25. C. P. Schnorr and G. Stumpf, A characterization of complexity sequences, Zeitschrift für Mathematische Logik und Grundlagen der Mathematik 21, 1 (1975), $47-56$.

26. M. Sipser, Halting space-bounded computations, Theoretical Computer Science 10, 3 (March 1980), 335-338.

27. C. H. Smith, A note on arbitrarily complex recursive functions, Notre Dame Journal of Formal Logic 29, 2 (Spring 1988), 198-207.

28. B. A. Trakhtenbrot, Complexity of algorithms and computations (in Russian), Course Notes, Novosibirsk University, Novosibirsk, Russia, 1967.

29. K. Wagner and G. Wechsung, Computational complexity, VEB Deutscher Verlag der Wissenschaften, Berlin, 1986.

30. P. Young, Easy constructions in complexity theory: Gap and speed-up theorems, Proceedings of the American Mathematical Society 37, 2 (February 1973), 555-563. 


\section{Index Terms}

abstract complexity, 14

additive-constant speedup, 3

Blum axioms, 2, 14

Blum complexity measure, 14

complexity class, 16

complexity hierarchy (see compression theorem)

complexity sequence (see prospective or realizable space complexity)

compression theorem, 3

computable operator, 7

computational complexity, 1

constructible space bound, 4

diagonalization, 4

effective operator (see computable operator)

effective speedup, 10

equivalent complexities, 12

equivalent program codes, 4

equivalent Turing machines, 3

fixed-point theorem, 4

fundamental theorem of complexity theory, 12,16

gap theorem, 7, 8, 16

Gödel number (see program code)

honest resource bound, 17

honesty theorem, 17, 18

isomorphism theorem, 13

iteration theorem (see s-m-n theorem)

leveling theorem, 11

limit-computable, 6

machine independence, 2, 13

machine-based complexity, 15, 16

mutual recursion, 4

naming theorem, 17

padding lemma, 5,13

parameter theorem (see s-m-n theorem)

program code, 4

program size, 1, 6

programming system, 13

prospective space complexity, 12

realizable space complexity, 12

recursion, 4

recursion theorem, 4,13

recursive-relatedness theorem, 15

s-m-n theorem, 13

space complexity, 1, 2

speedup theorem, 8,16

subconstructible space bound, 7,8

time complexity, 1 
trade-off between space and program size, 2, 6

Turing machine, 2

union theorem, 17

universal Turing machine, 6 